\title{
BMP et cancer
}

\section{Le Yin et le Yang des cellules souches}

> Dans le contexte normal, les bone morphogenetic proteins (BMP), membres de la superfamille du TGF $\beta$ (transforming growth factor $\beta$ ), sont des protéines clés dans la régulation des cellules souches adultes. Ces molécules influencent l'ensemble des propriétés fonctionnelles et phénotypiques (autorenouvellement, prolifération, différenciation, apoptose, quiescence, etc.) de ces cellules. Elles le font en agissant soit directement sur la cellule souche, soit par l'intermédiaire de son microenvironnement, et participent à l'équilibre entre les cellules souches et leur « niche ». Dans le contexte tumoral, l'altération de la voie des BMP déstabilise ces interactions et participe ainsi aux différentes étapes du processus de transformation. <

\section{Les BMP et leurs voies de signalisation}

Les bone morphogenetic proteins (BMP), membres de la superfamille du transforming growth factor (TGF) $\beta$, ont d'abord été caractérisées par leur capacité à induire la formation de structures osseuses in vivo et sont actuellement très utilisées en chirurgie orthopédique. À ce jour, une vingtaine de BMP ont été identifiées et leur implication démontrée dans un nombre impressionnant de processus biologiques dans de nombreux tissus, aussi bien lors du développement embryonnaire que chez l'adulte. La voie BMP est aussi impliquée dans le cancer ou l'ostéoporose, mais aussi dans des pathologies touchant le cœur, le poumon, les yeux et les organes de la reproduction. II est donc clair que cette famille unique et incontournable de protéines joue un rôle majeur dans les processus physiologiques et pathologiques.

Les BMP sont principalement sécrétées par les cellules stromales de l'environnement tissulaire. Ces molécules forment des homo- ou hétérocomplexes qui permettent la transduction de signaux à l'intérieur de la cellule. La voie principale de signalisation des BMP, appelée voie canonique, fait intervenir des récepteurs de type II à activité sérine/thréonine kinase (BMPRII)

\section{Karen Sagorny, Marion Chapellier, Bastien Laperrousaz, Véronique Maguer-Satta}

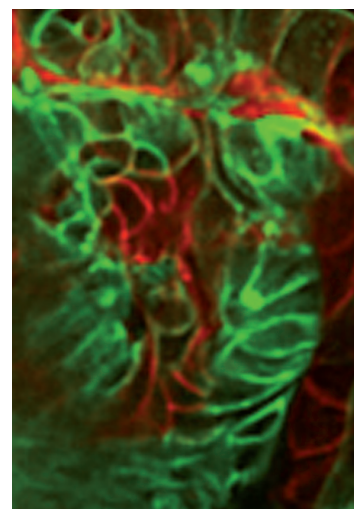

sur lesquels se fixe le ligand, et des récepteurs de type I (BMPRI) déterminant la spécificité du dimère. Trois récepteurs différents de type II (BMP receptor II [BMPRII], activin receptor II [ACTRIIA et ACTRIIB]), et trois récepteurs de type I (aussi nommés activin receptor-like kinase [ALK] : BMPRIa [ALK 3], BMPRIb [ALK6] et ACTRIa [ALK2]) ont été identifiés. La phosphorylation du BMPRI par le BMPRII déclenche la cascade de phosphorylation des effecteurs Smad (small mothers against decapentaplegic homolog) 1, 5 et 8, la formation d'un complexe avec Smad4 qui se relocalise dans le noyau et agit comme facteur de transcription sur des gènes cibles. Récemment, plusieurs voies indépendantes des protéines Smad, nommées voies non canoniques, ont été impliquées dans la signalisation des BMP et font par exemple intervenir les MAPK (mitogen activated protein kinases). Ces voies de signalisation sont finement contrôlées par un ensemble de régulateurs solubles, membranaires et cytoplasmiques (Figure 1). Des études récentes démontrent également que la voie BMP est régulée par des mécanismes épigénétiques d'hypo- et hyperméthylation des promoteurs, ainsi que par l'intermédiaire de microARN [1]. Enfin, un nombre croissant de protéines adaptatrices et des interactions avec d'autres voies de signalisation ont été mis en évidence, ce qui démontre l'importance et la complexité de la voie des BMP.

\section{BMP et cellules souches adultes: double compétence}

À l'heure actuelle, l'implication des BMP dans la biologie des cellules souches (CS) est clairement établie pendant la période embryonnaire et commence à être décryptée aussi chez l'adulte, en particulier chez l'homme. En effet, des études récentes ont permis de mettre en évidence de façon remarquable le rôle clé des BMP dans la régulation à la fois des CS tissulaires et des CS de la niche. 


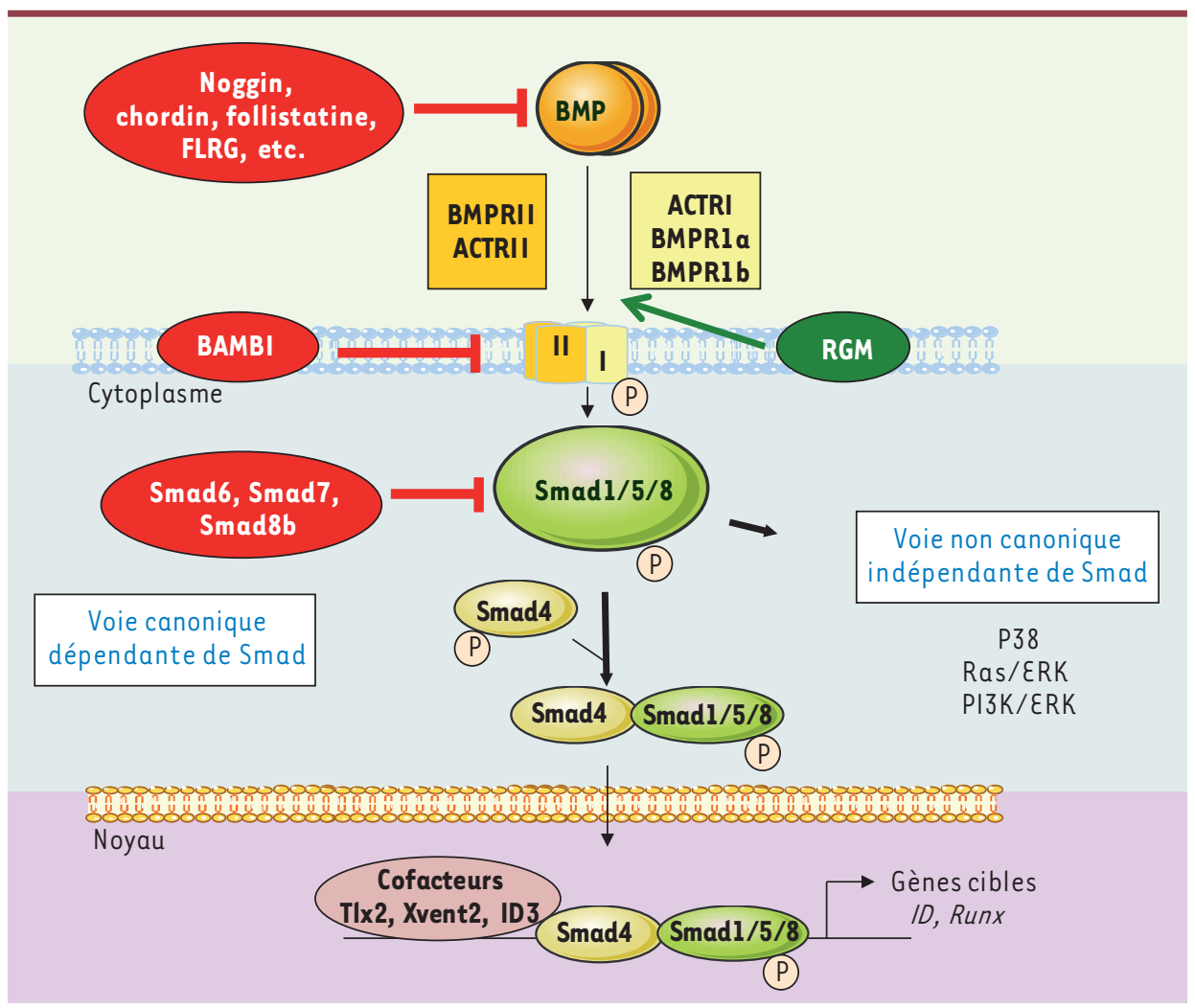

Figure 1. Voie de signalisation des BMP. Dans la voie dite canonique faisant intervenir les Smad, les BMP forment des dimères qui interagissent avec des tétramères de récepteurs membranaires de type sérine thréonine kinase (deux récepteurs de type I et deux récepteurs de type II). Après phosphorylation des récepteurs de type II, le signal est transmis à l'intérieur de la cellule par l'intermédiaire des protéines Smadl, 5 et 8 , phosphorylées à leur tour, et qui s'associent ensuite à Smad4 pour rejoindre le noyau et permettre la transcription de gènes cibles tels que les familles de gènes / $d$ et Runx. Cette voie de signalisation est finement régulée, et ceci de manière complexe par des protéines extracellulaires (noggin, chordin ou follistatine), membranaires (BAMBI [BMP and activin membrane-bound inhibitor], RGM [repulsive guidance molecule]) ou intracellulaires (Smad6, Smad7 et Smad8b). En plus de cette voie de signalisation classique, les effets des BMP peuvent être relayés par des voies non canoniques, indépendantes des Smad et faisant notamment intervenir les MAP kinases.

Rappelons que les cellules souches (CS) sont des cellules indifférenciées qui persistent chez l'adulte et jouent un rôle clé dans le maintien de l'homéostasie tissulaire grâce notamment à leur capacité à s'autorenouveler. En effet, par division asymétrique, une CS peut générer, d'une part une cellule fille qui va s'engager dans le processus de différenciation et, d'autre part, une cellule identique à la cellule d'origine qui persistera au sein du tissu adulte. Ces CS sont localisées dans des structures particulières appelées «niches » [31] avec lesquelles elles interagissent en permanence, via des contacts cellulaires et des molécules solubles sécrétées. Ce concept de niche a émergé en 1978 avec les travaux du groupe de Schofield qui ont mis en évidence l'existence de «zones spatialement bien définies, capables de fournir les facteurs nécessaires à la survie et au développement des CS, permettant la régénération des tissus dans les organismes adultes ». Outre son rôle de protection physique, la niche joue un rôle majeur dans le maintien de l'équilibre entre l'autorenouvellement et la différenciation des CS.

\section{Action directe des BMP}

sur les cellules souches

\section{Hétérogénéité de l'action des BMP sur les cellules souches selon l'espèce et le tissu}

Les BMP transduisent un signal à l'intérieur des CS, modulant ainsi leurs propriétés fonctionnelles (autorenouvellement, prolifération, différenciation, apoptose, quiescence, etc.). Les effets observés sont d'autant plus complexes qu'ils varient selon les systèmes étudiés et les BMP impliquées.

Ainsi, alors que chez l'homme BMP4 favorise in vitro l'autorenouvellement des CS hématopoïétiques (CSH), ce que démontre sa capacité à augmenter l'activité de repopulation des CSH chez des souris immunodéficientes NOD/ SCID (non obese diabetic) severe combined immunodeficient) irradiées [2, 32], cette protéine a un effet différent dans d'autres types de CS. Par exemple, toujours chez l'homme, dans le système nerveux, le traitement par BMP4 induit l'arrêt des CS neurales (CSN) en phase GO/Gl du cycle cellulaire. Cet arrêt est réversible et les cellules conservent leurs propriétés de CS [3]. Le maintien de la quiescence de ces CSN fait intervenir le récepteur BMPRIa (ALK3) et Smad4 en réponse à BMP2 et BMP4 (Figure 2). Les BMP sont donc capables d'induire la différenciation, l'autorenouvellement ou la quiescence des CS, selon le contexte cellulaire mais également l'espèce impliquée. En effet, dans le système murin, BMP4 permet, non pas la quiescence, mais l'engagement vers la différenciation des CSN, la perte de leur potentiel souche et l'acquisition de propriétés caractéristiques de cellules plus matures [4]. La voie BMP est aussi capable de maintenir la quiescence de certaines CS dans le tissu épithélial, chez la souris : elle régule ainsi l'autorenouvellement des CS 


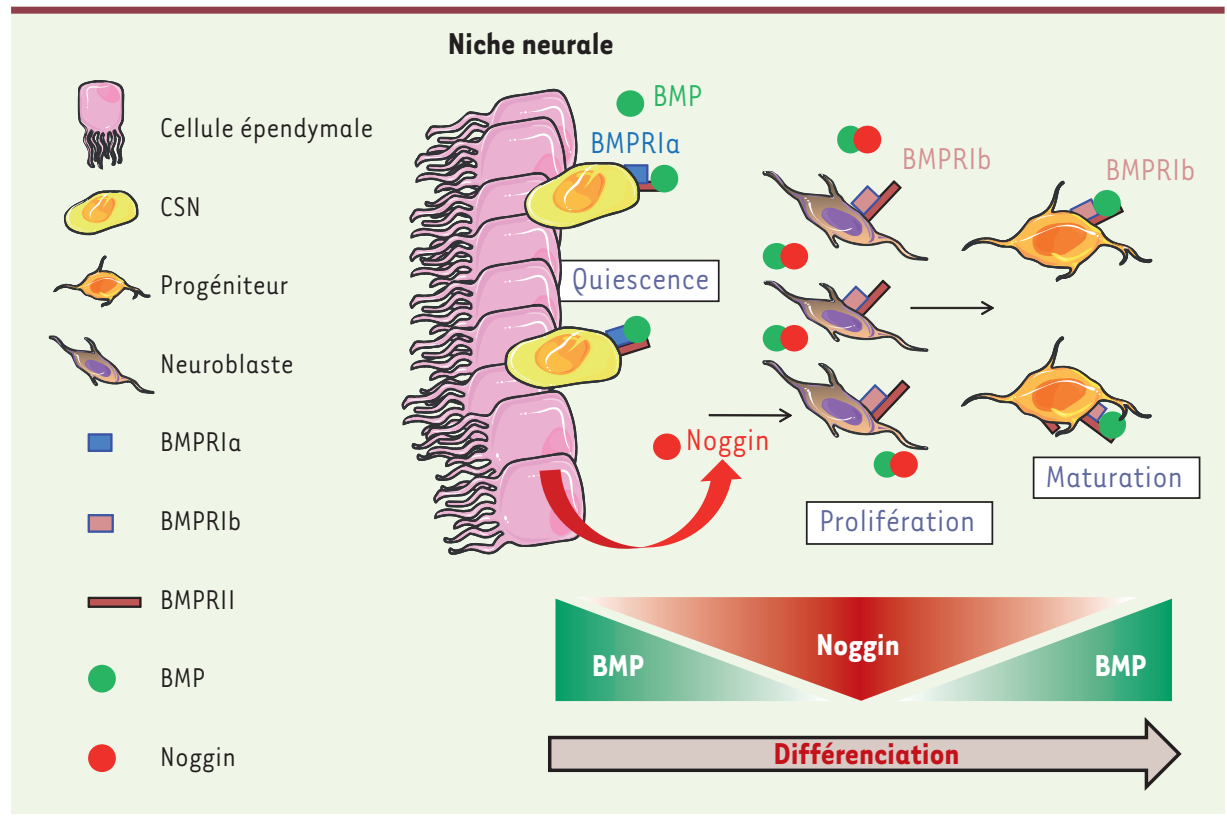

Figure 2. Dualité fonctionnelle des BMP dans le système neural. Dans le système neural, les BMP régulent la quiescence des CSN (cellules souches neurales) via le récepteur BMP de type la (BMPRIa), mais induisent la différenciation et la maturation des neurones via le récepteur BMP de type lb (BMPRIb) exprimé par les progéniteurs et les neuroblastes. Ces effets complexes sur la différenciation neurale sont régulés spatialement par les cellules épendymales de la niche qui sécrètent l'inhibiteur Noggin, créant ainsi un gradient de signalisation BMP. L'inhibition des signaux BMP va permettre aux CSN de quitter le stade quiescent pour proliférer puis se différencier. Enfin, lorsque les cellules en cours de différenciation migrent à la périphérie de la niche, la concentration en Noggin diminue et le signal BMP redevient alors actif, permettant la maturation des neurones.

folliculaires [5] et intestinales [6] via la voie Wnt/ $\beta$-caténine. BMP4 favoriserait également la survie des CS et le maintien de leur multipotence [7] dans le système mésenchymateux. Ces exemples illustrent la complexité à la fois des effets biologiques des BMP et de la spécification du signal, dont la résultante biologique est l'intégration de l'ensemble de ces paramètres.

\section{BMP et induction de l'engagement des cellules souches vers le processus de différenciation}

Un certain nombre d'études ont également mis en évidence un effet des BMP sur la différenciation des CS en progéniteurs. Dans le système hématopoïétique humain, selon les molécules présentes dans l'environnement, BMP4 oriente les CSH vers les lignages mégacaryocytaire ou érythrocytaire, en synergie avec l'activine A, l'érythropoïétine (EP0) ou le SCF (stem cell factor) [8,9]. De même, les BMP influencent la différenciation des cellules stromales mésenchymateuses (CSM) vers différents lignages : adipocytaire, ostéogénique ou encore chondrocytaire [10]. Dans le pancréas, le traitement par BMP4 de cellules pluripotentes (lignée AR42J de carcinome pancréatique de rat) induit leur prolifération puis leur différenciation [11]. L'injection d'un anticorps neutralisant BMP4 dans des souris IFN $\gamma^{\top-N O D}$ (souris NOD déficientes en interféron $\gamma$ qui représentent un modèle de régénération pancréatique) réduit l'expansion des progéniteurs pancréatiques. BMP4 semble donc contrôler à la fois l'expansion et la différenciation terminale des progéniteurs du pancréas. Enfin, dans le système neural, les gradients de concentration de BMP4 conditionnent le choix du lignage cellulaire dans lequel s'engagent les CSN, suggérant une régulation spatiale de l'action de BMP4 sur la neurogenèse et une dépendance vis-à-vis des signaux environnementaux [12, 13] (Figure 2). Ainsi, les BMP sont des inducteurs conditionnels de l'engagement des CS dans les voies de différenciation spécifiques, dont l'action finale dépend de la présence de cofacteurs spécifiques.

Les BMP peuvent donc induire le maintien de la multipotence, ou, inversement, la différenciation des CS. L'une des grandes difficultés de l'analyse des effets des BMP dans la biologie des CS réside donc dans cette multiplicité d'effets biologiques souvent apparemment contradictoires. L'effet biologique est conditionné à la fois par des paramètres intrinsèques (éléments composant les voies de signalisation) et des paramètres extrinsèques comme la régulation spécifique du tissu par les éléments de l'environnement. Ainsi, de l'embryogenèse jusqu'à l'âge adulte, les BMP agissent sous le contrôle très étroit de l'environnement cellulaire sur une multitude de systèmes (Figure 3 ).

Action des BMP sur la niche environnementale des CS Quel que soit le système étudié, les cellules souches résident dans des niches constituées d'une multitude de types cellulaires qui synthétisent de nombreux composés dont les protéines BMP. Celles-ci, en retour, sont capables d'agir sur les différents éléments et acteurs de cette niche qui, à leur tour, modulent les CS : ainsi se crée un réseau de régulation dynamique réciproque en dialogue perpétuel.

- La niche médullaire contient les éléments de la matrice extracellulaire et différents types cellulaires dont les CSM, à l'origine des ostéoblastes, chondrocytes et adipocytes. L'utilisation de souris déficientes en BMPRIa a, pour la première fois, apporté la preuve de l'action indirecte de 


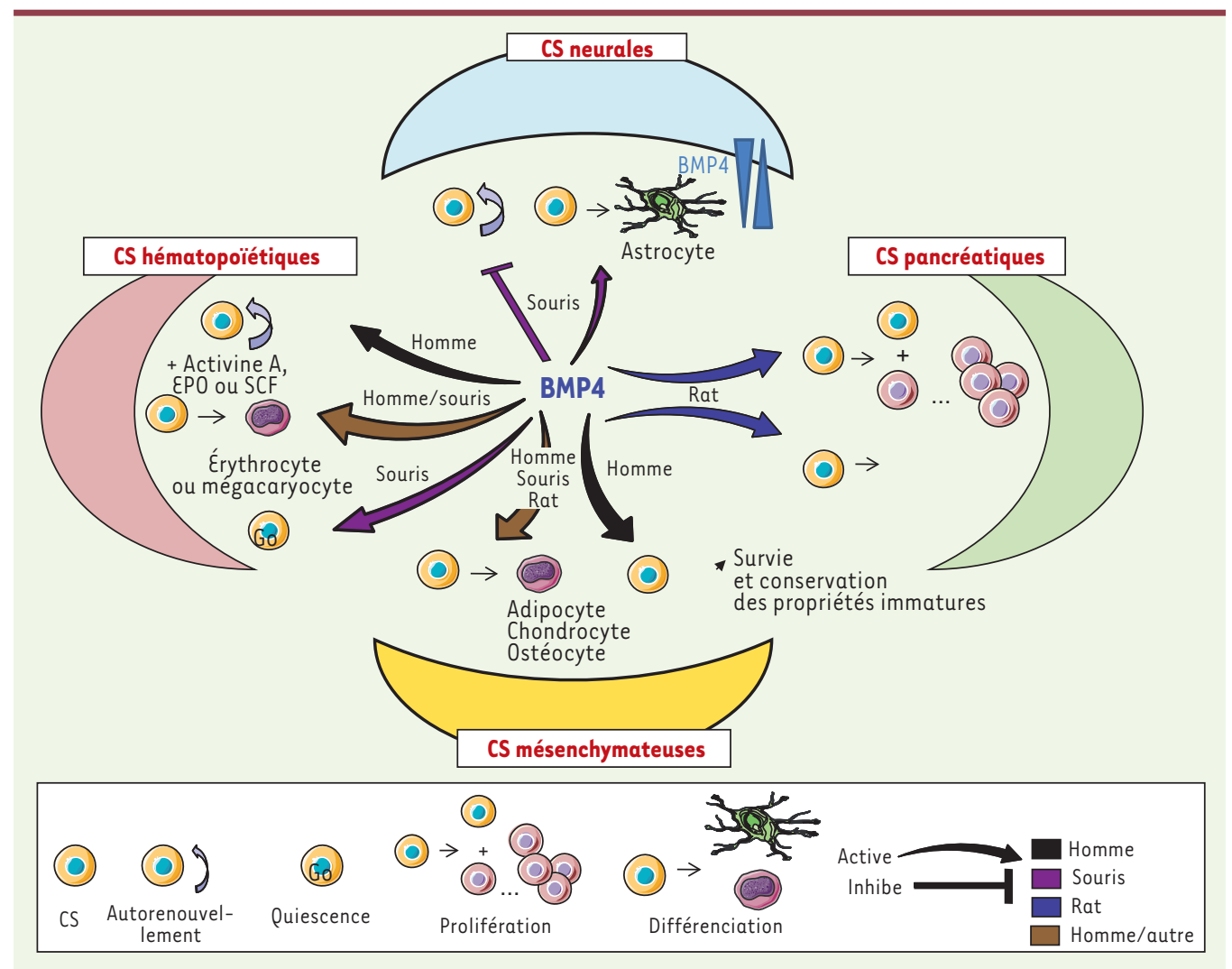

Figure 3. La protéine BMP 4 : une pléiade d'effets sur les CS adultes. BMP4 à elle seule est capable de réguler les différents types de CS adultes avec des effets différents selon l'espèce considérée. Sur un même type de CS adulte et dans une espèce donnée, BMP4 peut également induire des effets différents en fonction de sa concentration ou encore de ses partenaires locaux.

la famille BMP sur le compartiment des CSH via l'environnement : chez ces souris, l'augmentation du nombre d'ostéoblastes dans la moelle des souris $\mathrm{BMPRIa}^{-/-}$conduit à une expansion significative des CSH transplantées dans ces animaux [14]. Cette étude démontre de façon remarquable que les molécules de la famille BMP contrôlent la taille de la niche hématopoïétique par l'intermédiaire des ostéoblastes, support direct des propriétés des CSH.

- Dans la zone sous-ventriculaire du cerveau (région où se localisent les CS neurales), les CS - qui expriment des caractéristiques astrocytaires -, les progéniteurs et les neuroblastes jouxtent les cellules épendymales qui bordent l'ensemble des cavités ventriculaires de cette zone. Dans cette région, les cellules épendymales sécrètent noggin, un inhibiteur majeur de BMP4. La neutralisation de BMP4 favorise la neurogenèse via la prolifération et l'engagement vers la différenciation neurale des CSN, alors que dans le striatum par exemple, la sécrétion de BMP4 par les cellules environnantes favorise au contraire la différenciation astrocytaire des CSN [15] (Figure 2). Ainsi, la régulation spatiale de la signalisation BMP établie dans le cerveau adulte par le microenvironnement contrôle la différenciation des CS neurales. - Dans le système épithélial cutané, lors de la régénération du follicule pileux, les CS migrent du bourgeon vers la matrice et reposent sur les cellules de la papille dermique responsables de l'induction de la formation d'un nouveau follicule [16]. Les cellules de la gaine dermique sécrètent l'inhibiteur noggin tandis que les cellules de la papille dermique synthétisent des BMP dont BMP4. Un gradient d'activité BMP est ainsi formé : concentration faible de BMP à la base du follicule où la prolifération des cellules de la niche est favorisée, concentration forte en position plus distale qui réduit la prolifération et favorise la différenciation. II s'établit ainsi une boucle d'autorégulation complexe de l'activité de la protéine BMP4, similaire à celle de la niche neurale, dépendante à la fois des gradients de concentration de BMP4 et de celle de son inhibiteur noggin. L'inactivation du récepteur BMPRIa dans les cellules de la papille dermique a démontré le rôle de la voie des BMP dans l'induction d'un nouveau cycle folliculaire.

- Le système intestinal murin, un autre exemple d'épithélium au renouvellement rapide, a été particulièrement bien analysé. L'épithélium intestinal est une monocouche de cellules reposant sur une membrane basale et formant des invaginations : les villosités (vers la lumière intestinale) et les cryptes (qui s'enfoncent dans la muqueuse) [33]. Les villosités sont constituées principalement de cellules différenciées qui assurent des fonctions spécialisées et dont le renouvellement est assuré par des CS logées au fond des cryptes (2 à 5 CS par crypte). L'inhibition conditionnelle de BMPRla induit l'expansion des CS et des progéniteurs intestinaux, ce qui confirme le rôle de la signalisation en aval de ce récepteur dans le maintien de CS indifférenciées [6]. L'inhibition de la voie BMP chez des souris transgéniques exprimant noggin induit la formation ectopique de 
cryptes [17], lieu de résidence des CS intestinales. De plus, d'autres travaux ont démontré que la voie BMP inhibe la signalisation Wnt [6], elle-même impliquée dans la régulation de l'autorenouvellement des CS, soulignant à nouveau la complexité de la signalisation BMP.

La voie BMP est donc directement impliquée dans le contrôle des CS et de leur niche. En effet, la distribution et l'action des BMP dans le temps et l'espace sont dictées par la niche elle-même sous le contrôle de la voie des BMP (composition et taille) [14]. L'équilibre entre ces différents paramètres permet une régulation fine de la biologie des CS au sein de leur microenvironnement.

\section{BMP et processus oncogénique}

Et dans le cancer? L'implication de la famille BMP dans des processus pathologiques a été documentée, en particulier dans le cancer. Bien que son rôle majeur dans les processus oncogéniques soit indéniable, ce n'est que très récemment que son implication dans la biologie des CS cancéreuses (CSC) a été suspectée.

\section{BMP et cellules souches cancéreuses (CSC)}

L'existence de CSC a pour la première fois été démontrée de façon formelle dans des leucémies aiguës myéloïdes (LAM) [18]. Cette découverte repose sur l'observation qu'une sous-population de cellules leucémiques, de phénotype $\operatorname{CD} 34^{+} \operatorname{CD} 38^{-}$, est seule capable de générer des cellules cancéreuses après son injection à des souris NOD/ SCID. On appelle CSC une fraction minoritaire au sein de la tumeur qui a conservé - ou acquis - les propriétés d'autorenouvellement et de prolifération caractéristiques des CS normales, et capable à elle seule de reconstituer l'ensemble des différentes populations qui composent la tumeur.

Bien que ce concept soit encore largement discuté, des travaux ont depuis mis en évidence l'existence de telles sous-populations dans les glioblastomes, les mélanomes, les cancers du côlon et du sein [19]. En clinique, cette découverte majeure prédit que si l'éradication des cellules tumorales induit une rémission, seule la destruction des CSC conduirait à une guérison.

Les BMP sont des régulateurs clés de la biologie des CS normales, mais qu'en est-il de leur rôle dans les CSC ? À ce jour, les principaux travaux qui démontrent l'implication des BMP dans la régulation des CSC concernent le système neural et la peau. Dans le glioblastome, BMPRIb serait impliqué dans le maintien du phénotype des CSC [20]. En effet, au sein de la tumeur, il existe une fraction cellulaire réfractaire à la différenciation induite par BMP4 qui persiste et favorisera la reconstitution de la tumeur. Dans cette fraction de cellules cancéreuses immatures, l'expression de BMPRIb est éteinte par la méthylation de son promoteur et sa réexpression en réponse à un agent déméthylant permet de restaurer la différenciation des CSC sous l'effet de BMP4. Ainsi, le traitement des CSC neurales par BMP4 diminue leur prolifération sans affecter leur viabilité, induit leur différenciation en précurseurs neuraux et diminue leur capacité à induire la formation de tumeurs dans des souris NOD/SCID. BMP4 réduit le pool de CSC neurales en les poussant vers un processus de différenciation [21] et constituerait donc un agent thérapeutique potentiel dans cette pathologie particulièrement agressive.

Dans le mélanome, l'existence de CSC a été mise en évidence en 2006 chez l'homme, même si cette notion a depuis été contestée [34]. Ces CSC expriment des marqueurs spécifiques tels que ABCB5 (ATP-binding cassette sub-family $B$ member 5 ) et surexpriment BMPRIa [19]. Cependant, l'implication fonctionnelle de la voie BMP dans les CSC de mélanomes n'a pas encore été décrite.

L'étude du rôle des BMP dans la régulation des CSC ne fait que commencer et la régulation indirecte des CSC via leur niche environnementale reste largement inexplorée.

\section{BMP et microenvironnement des CSC}

À l'heure actuelle, un grand nombre d'études tendent à démontrer que le microenvironnement des CSC serait altéré sans être formellement transformé. Dans les leucémies, certains travaux suggèrent que l'altération pourrait toucher à la fois les populations cellulaires de ce microenvironnement et les composants de la matrice extracellulaire comme les intégrines, certaines cytokines, la fibronectine ou encore les ligands de la voie Wnt [22-24]. L'altération du microenvironnement a également été rapportée dans les tumeurs solides. Elle se traduit par une désorganisation des fibres de collagène et de fibronectine induisant une rigidité accrue du tissu, un regroupement de certaines intégrines ainsi que l'activation anormale de certaines voies de signalisation [25].

La majorité des hémopathies malignes sont caractérisées par une altération plus ou moins marquée de la composition de la moelle osseuse, avec le plus souvent une myélofibrose qui s'accentue avec la progression de la maladie [26]. Or les BMP sont connues pour leur implication dans les processus ostéogéniques. II n'est donc pas exclu qu'une dérégulation de la voie BMP, matérialisée par exemple par une altération des concentrations de BMP solubles dans la moelle osseuse, puisse être impliquée dans cette myélofibrose [27]. De manière intéressante, différents travaux indiquent que l'Imatinib, traitement de première intention des leucémies myéloödes chroniques (LMC), augmenterait in vitro l'expression transcriptionnelle de BMP2 dans des CSM humaines [28] et rétablirait une structure médullaire cohérente [29].

Par ailleurs, le cancer du sein est souvent associé au développement de métastases osseuses. La présence de microcalcifications a été mise en évidence dans la glande mammaire et constitue un important marqueur diagnostique. Une étude à partir des cellules issues de 


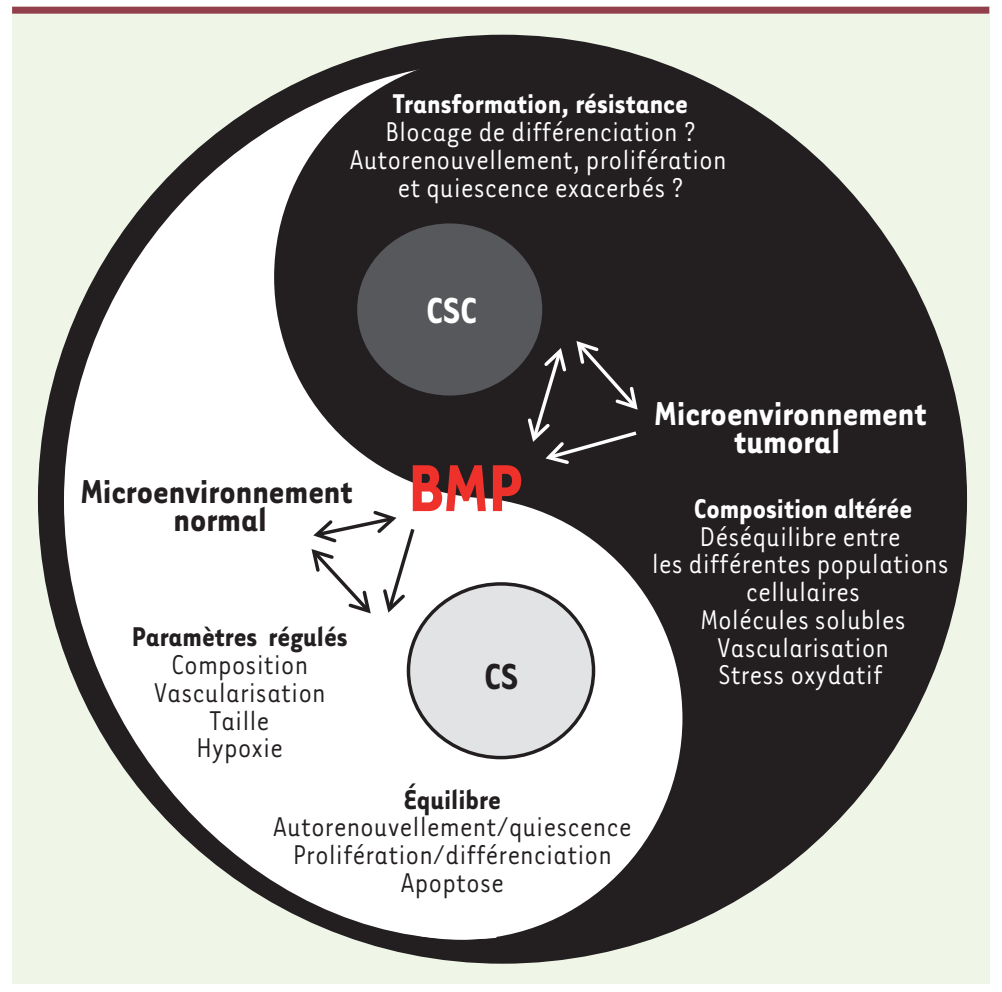

Figure 4. BMP et cancer: le Yin et le Yang des CS. Les BMP sont des régulateurs clés de la biologie des CS agissant sur l'ensemble de leurs propriétés fonctionnelles. Ces molécules permettent ainsi le maintien de leur homéostasie au sein du microenvironnement. En revanche, dans un contexte tumoral, ces mêmes molécules participent à la dérégulation de la CS ainsi que de son microenvironnement, conduisant au phénotype de CS cancéreuse et attestant de leur implication dans toutes les étapes du processus de transformation tumorale.

phenotypic properties of the stem cell population (self-renewal, proliferation, differentiation, apoptosis, quiescence, etc.). They can act directly on the stem cell or through its microenvironment, contributing to the tight balance of this system. In the tumorigenic context, alterations of the BMP signalling are involved in the deregulation of the interaction between stem cells and their microenvironment and, as such, participate to the different steps of the transformation process. $\diamond$

tumeurs mammaires de rats a mis en évidence que l'expression de BMP2 serait suffisante au déclenchement du processus de formation de ces microcalcifications [30]. Plusieurs études rapportent également des altérations d'expression des BMP dans le microenvironnement mammaire. Malgré ces observations, à l'heure actuelle, le lien entre les BMP, l'environnement et la transformation cellulaire n'a pas encore été établi.

\section{Conclusion et perspectives cliniques}

Dans le contexte normal, les BMP régulent l'ensemble des propriétés fonctionnelles et phénotypiques des CS. Les effets sont soit directs, soit via le microenvironnement qui, à son tour, régule les CS. II existe donc un véritable et perpétuel dialogue entre les CS et leurs partenaires locaux, contrôlé en partie par les membres de la famille BMP. Dans le contexte tumoral où il existe une dérégulation à la fois des CS et de leur microenvironnement, la voie BMP apparaît donc comme un élément clé du processus de transformation maligne (Figure 4). Ainsi, il s'avère primordial de continuer à étudier et à déchiffrer les mécanismes d'action des BMP dans la régulation des CS normales. Ceci permettra vraisemblablement de mieux appréhender les rouages de l'émergence, du maintien et des propriétés fonctionnelles des CSC, sur lesquelles la voie BMP exerce un double pouvoir. $\diamond$

\section{SUMMARY}

\section{BMP and cancer: the Yin and Yang of stem cells}

In a normal context, bone morphogenetic proteins (BMPs), members of the TGF $\beta$ superfamily, are key players in adult stem cell biology. They are involved in the control of the overall functional and

\section{CONFLIT D'INTÉRÊTS}

Les auteurs déclarent n'avoir aucun conflit d'intérêts concernant les données publiées dans cet article.

\section{RÉFÉRENCES}

1. Kimura K, Toyooka S, Tsukuda K, et al. The aberrant promoter methylation of BMP3b and BMP6 in malignant pleural mesotheliomas. Oncol Rep 2008; 20 : 1265-8.

2. Bhatia M, Bonnet $D$, Wu D, et al. Bone morphogenetic proteins regulate the developmental program of human hematopoietic stem cells. J Exp Med $1999 ; 189: 1139-48$.

3. Mira $\mathrm{H}$, Andreu Z, Suh $\mathrm{H}$, et al. Signaling through BMPR-IA regulates quiescence and long-term activity of neural stem cells in the adult hippocampus. Cell Stem Cell $2010 ; 7: 78-89$

4. Bonaguidi MA, McGuire T, Hu M, et al. LIF and BMP signaling generate separate and discrete types of GFAP-expressing cells. Development 2005 ; $132:$ 5503-14.

5. Kobielak K, Stokes N, de la Cruz J, et al. Loss of a quiescent niche but not follicle stem cells in the absence of bone morphogenetic protein signaling. Proc Natl Acad Sci USA 2007 ; 104 : 10063-8.

6. He XC, Zhang J, Tong WG, et al. BMP signaling inhibits intestinal stem cell self-renewal through suppression of Wnt-beta-catenin signaling. Nat Genet $2004 ; 36$ : 1117-21.

7. Vicente Lopez MA, Vazquez Garcia MN, Entrena A, et al. Low doses of bone morphogenetic protein 4 increase the survival of human adipose-derived stem cells maintaining their stemness and multipotency. Stem Cells Dev $2011 ; 20: 1011-9$

8. Maguer-Satta V, Bartholin L, Jeanpierre S, et al. Regulation of human erythropoiesis by activin A, BMP2, and BMP4, members of the TGFbeta family. Exp Cell Res $2003 ; 282$ : 110-20.

9. Jeanpierre $S$, Nicolini FE, Kaniewski B, et al. BMP4 regulation of human megakaryocytic differentiation is involved in thrombopoietin signaling. Blood 2008 ; 112 : 3154-63.

10. Kang $Q$, Song WX, Luo $Q$, et al. A comprehensive analysis of the dual roles of BMPs in regulating adipogenic and osteogenic differentiation of mesenchymal progenitor cells. Stem Cells Dev 2009 ; 18 : 545-59.

11. Hua H, Zhang YQ, Dabernat S, et al. BMP4 regulates pancreatic progenitor cell expansion through Id2. J Biol Chem 2006 ; 281 : 13574-80. 


\section{RÉFÉRENCES}

12. Liu SY, Zhang ZY, Song $Y C$, et al. SVZa neural stem cells differentiate into distinct lineages in response to BMP4. Exp Neurol 2004 ; 190 : 109-21.

13. Rajan P, Panchision DM, Newell LF, McKay RD. BMPs signal alternately through a SMAD or FRAPSTAT pathway to regulate fate choice in CNS stem cells. J Cell Biol $2003 ; 161: 911-21$.

14. Zhang J, Niu C, Ye L, et al. Identification of the haematopoietic stem cell niche and control of the niche size. Nature $2003 ; 425: 836-41$.

15. Lim DA, Tramontin AD, Trevejo JM, et al. Noggin antagonizes BMP signaling to create a niche for adult neurogenesis. Neuron $2000 ; 28: 713-26$.

16. Rendl M, Polak L, Fuchs $\varepsilon$. BMP signaling in dermal papilla cells is required for their hair follicleinductive properties. Genes Dev $2008 ; 22: 543-57$.

17. Haramis AP, Begthel $H$, van den BM, et al. De novo crypt formation and juvenile polyposis on BMP inhibition in mouse intestine. Science 2004 ; 303 : 1684-6.

18. Bonnet $D$, Dick JE. Human acute myeloid leukemia is organized as a hierarchy that originates from a primitive hematopoietic cell. Nat Med 1997 ; $3: 730-7$.

19. Schatton T, Frank MH. Cancer stem cells and human malignant melanoma. Pigment Cell Melanoma Res $2008 ; 21: 39-55$.

20. Lee J, Son MJ, Woolard K, et al. Epigenetic-mediated dysfunction of the bone morphogenetic protein pathway inhibits differentiation of glioblastoma-initiating cells. Cancer Cell 2008; 13 69-80.

21. Piccirillo SG, Reynolds BA, Zanetti N, et al. Bone morphogenetic proteins inhibit the tumorigenic potential of human brain tumour-initiating cells. Nature $2006 ; 444: 761-5$.

22. Ruoslahti $\varepsilon$. Fibronectin and its integrin receptors in cancer. Adv Cancer Res $1999 ; 76: 1-20$.

23. Hideshima T, Bergsagel PL, KuehI WM, Anderson KC. Advances in biology of multiple myeloma: clinical applications. Blood $2004 ; 104: 607-18$

24. Simon M, Grandage VL, Linch DC, Khwaja A. Constitutive activation of the Wnt/beta-catenin signalling pathway in acute myeloid leukaemia. Oncogene $2005 ; 24: 2410-20$.

25. Paszek MJ, Zahir N, Johnson KR, et al. Tensional homeostasis and the malignant phenotype. Cancer Cell $2005 ; 8: 241-54$.
26. Buesche G, Ganser A, Schlegelberger B, et al. Marrow fibrosis and its relevance during imatinib treatment of chronic myeloid leukemia. Leukemia $2007 ; 21: 2420-7$.

27. Bock 0, Hoftmann J, Theophile K, et al. Bone morphogenetic proteins are overexpressed in the bone marrow of primary myelofibrosis and are apparently induced by fibrogenic cytokines. Am J Pathol 2008 ; 172 : 951-60.

28. Tibullo D, Giallongo C, La Cava $P$, et al. Effects of imatinib mesylate in osteoblastogenesis. Exp Hematol 2009 ; 37 : 461-8.

29. Thiele J, Kvasnicka HM, Schmitt-Graeff A, et al. Bone marrow changes in chronic myelogenous leukaemia after long-term treatment with the tyrosine kinase inhibitor STI571: an immunohistochemical study on 75 patients. Histopathology $2005 ; 46: 540-50$.

30. Liu F, Bloch N, Bhushan KR, et al. Humoral bone morphogenetic protein 2 is sufficient for inducing breast cancer microcalcification. Mol Imaging 2008 $7: 175-86$.

31. Lataillade JJ, Brunet de la Grange P, Uzan G, Le Bousse-Kerdilès MC. Les cellules souches ont-elles l'âge de leur niche ? À la recherche d'un sérum de jouvence. Med Sci (Paris) $2010 ; 26: 582-5$.

32. Cachat A, Villaudy J, Rigal D, Gazzolo L, Duc Dodon M. Les souris ne sont pas des hommes et pourtant... Ce que les souris humanisées nous apprennent sur les maladies infectieuses. Med Sci (Paris) 2012 ; $28: 63-8$.

33. Joubert D, Hollande F, Jay P, Legraverend C. Les cellules souches intestinales: 30 ans d'une histoire exemplaire. Med Sci (Paris) 2009 ; 25 : 441-4.

34. Häfner $S$, Coulombel L. L'oligarchie contestée des cellules souches cancéreuses. Med Sci (Paris) 2009 ; 25 : 227-8.

\section{TIRÉS À PART}

V. Maguer-Satta

\section{Collection SCIENCE ET BIOMÉDECINE}

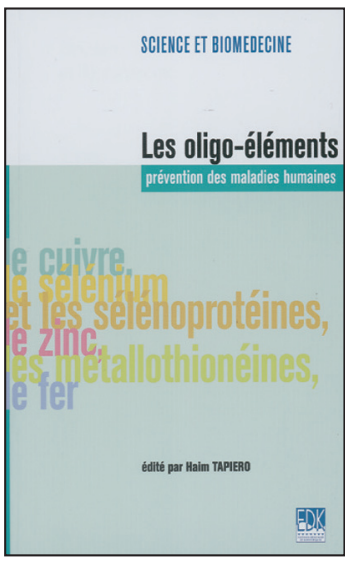

ISBN : 2-84254-107-3 64 pages

$10 €+3 €$ de port $=13 €$ TTC

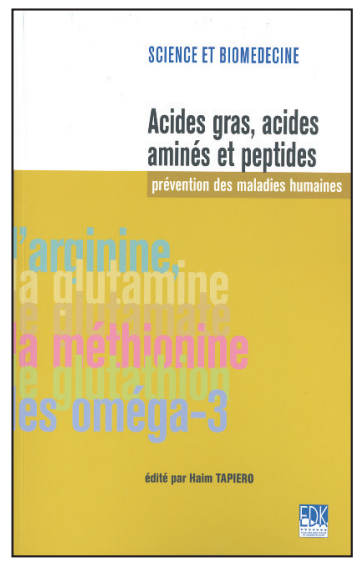

ISBN : 2-84254-108-1 80 pages $12 €+3 €$ de port $=15 €$ TTC

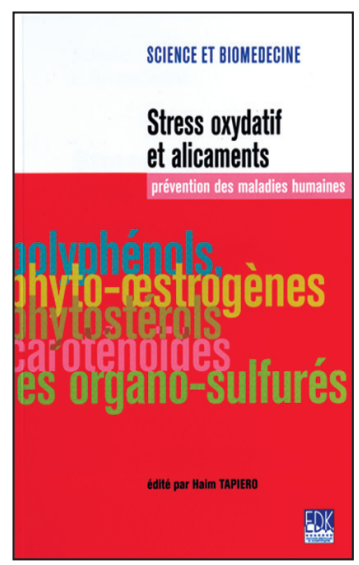

ISBN : 2-84254-111-1 86 pages $14 €+3 €$ de port $=\mathbf{1 7} €$ TTC

\section{Bon de commande}

À retourner à EDK, 25, rue Daviel - 75013 Paris

Tél. : 0158101905 - Fax : 0143293262 - E-mail : edk@edk.fr

NOM :

Prénom :

Adresse :

Code postal :

Ville :

Pays :

Fonction :

Je souhaite recevoir

soit un total de

$\square$ Par chèque, à l'ordre de $\mathbf{E} \mathbf{D} \mathbf{K}$

$\square$ Par carte bancaire : $\square$ Visa $\square$ Eurocard/Mastercard

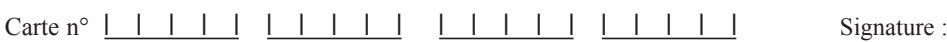

Date d'expiration: $\quad 1 \quad 1 \quad 111$

$N^{\circ}$ de contrôle au dos de la carte : $\quad$ । $\quad|\quad|$ 